\title{
Reliability Acceptance Criteria for Deteriorating Elements of Structural
}

\section{Systems}

\author{
Daniel Straub $^{1}$ \& Armen Der Kiureghian ${ }^{2}$
}

\begin{abstract}
A systematic approach to determining reliability-based acceptance criteria for deteriorating elements in structural systems is proposed, as a basis for calibration of safety factors in codes and standards and for verifying acceptability of inspection and maintenance strategies for specific structures. The goal is to establish deterioration acceptance criteria for the elements of a structural system in compliance with criteria formulated for the system. Existing methods significantly overestimate the deterioration reliability of redundant structural systems because they neglect the joint effect of deterioration failures of different elements. To more realistically capture the load-sharing behavior of deteriorating redundant structural systems, it is proposed to establish deterioration acceptance criteria based on easily computable, idealized structural systems, which are calibrated to the characteristics of the real structure. The approach is validated on an example structural system and is found to represent a significant improvement over current methods. The paper concludes with a study of the main factors influencing acceptance criteria of deterioration reliability.
\end{abstract}

\footnotetext{
${ }^{1}$ Associate Professor, Engineering Risk Analysis Group, Technical University Munich, Arcisstr. 21, 80290 München, Germany. Email: straub@tum.de

${ }^{2}$ Taisei Professor of Civil Engineering, Dept. of Civil \& Environmental Engineering, Univ. of California, Berkeley, CA 94720. Email: adk@ce.berkeley.edu
} 


\section{Introduction}

Owners of structural systems are confronted with the problem of determining whether their structures and their inspection/maintenance/repair policies are acceptable with regard to potential deterioration failures. While this applies equally to newly built and existing structures, the problem is particularly relevant for the latter, for which the cost of increasing the reliability is generally much higher (Melchers 2001). Extensive research has been carried out on probabilistic modeling of deterioration in structural systems, as reviewed by Frangopol et al. (2004). Furthermore, methods for reliability analysis of deteriorating structural systems have been developed over the past two decades, including works by Mori and Ellingwood (1993), Li (1995), Ciampoli (1998), Estes and Frangopol (1999) and Stewart and Val (1999). These methods enable the computation of the time-variant reliability of structures with deteriorating elements, which in the general case is a highly complex task. Because of this complexity, such integrated reliability analysis is rarely performed in engineering practice; rather, deterioration is assessed at the level of structural details or elements. The present paper, therefore, follows a different strategy. It proposes a method for determining the required level of deterioration reliability at the level of structural elements that ensures acceptability of the risks at the structural system level. The method accounts for the relevant influencing factors in an approximate sense, while remaining sufficiently simple for practical applications. The approach is motivated by practices in structural engineering for fixed offshore structures applied since the 1980s, where acceptance criteria for fatigue deterioration are determined as a function of the structural redundancy with respect to element failure (Kirkemo 1990, Moan 2005). The proposed method can be applied to determine acceptability of specific structures and 
inspection/maintenance strategies (Straub and Faber 2005b), or for calibration of safety factors for deterioration limit states in codes and standards.

\section{Problem setting}

Existing codes typically specify design criteria and safety factors for individual structural elements. This applies for failures caused by static or dynamic overloading of the structural elements, as described by ultimate limit states, as well as for deterioration failures, e.g., described by fatigue limit states. However, deterioration failures exhibit some fundamental differences as compared to overloading failures, which make it necessary to explicitly account for the system characteristics. When structural systems collapse because of overloading, all elements involved in the realized failure mode normally fail during the same load event (when considering cascading failure sequences as a single event). For this reason, the safety margins of the individual elements exhibit strong statistical dependence and the system reliability approximately equals the reliability of the individual elements (assuming that all elements have been designed to have the same target reliability index). Failures of structural elements caused by deterioration, on the other hand, are likely to occur at different times depending on the nature of the deterioration process. These events have lower statistical dependence and the corresponding system reliability, therefore, substantially differs from the deterioration reliability of the individual elements. For these reasons, the acceptability of deterioration failures must be assessed as a function of structural redundancy. In addition, deterioration can be detected before failure occurs, but deterioration failures can also remain undetected. The inspection and repair policies, therefore, influence the acceptability of deterioration failures. These aspects are partly reflected in design codes such as Eurocode 3 (1992) and NORSOK (1998), where safety factors for fatigue limit states are specified as a function of the 
consequences of element failure (structural importance) and the possibility to inspect an element.

In the past, reliability-based acceptance criteria for deterioration limit states have been considered mainly for structures subject to fatigue, in particular fixed offshore structures, e.g., HSE (2002), Ronalds et al. (2003), Moan (2005), and Straub and Faber (2005a). These acceptance criteria were determined as a function of the structural importance of the considered element. The structural importance of each element was assessed by comparing the overall capacity of the intact structural system with the capacity of the structural system when the element is removed. As shown in this paper, this approach is only suitable for elements with high reliability and if the statistical dependence among deterioration failures is low, because it neglects the possibility of joint occurrence of more than one deterioration failure. These conditions often are not satisfied in practice. For many structural systems, deterioration states of structural elements are correlated. As previously stated, this correlation is lower than that for limit states of element failures due to overloading (for which the correlation coefficient is close to one), but it is non-negligible for most structural systems. As an example, in an investigation of the integrity of mooring systems for floating offshore structures, it was found that deterioration typically affects all mooring lines to roughly the same extent (HSE 2006), which implies large statistical dependence among the corresponding deterioration states. Since mooring systems have significant redundancy (failure of an individual mooring line is generally not critical), the dependence among the deterioration processes of different elements strongly influences the system reliability. Another example is the study reported by Vrouwenvelder (2004), which inferred statistical dependence among fatigue performance of welded joints by comparing within-batch variability to batch-to-batch variability. On this basis, the correlation coefficient between fatigue crack growth parameters of two welded joints in the 
same structure was estimated as 0.85 . Thus, fatigue failure events in structural systems are expected to exhibit significant statistical dependence, which must not be neglected.

The goal of this paper is to introduce a practical yet scientifically sound method for determining reliability acceptance criteria for deteriorating elements in general structural systems, as a function of overall system acceptance criteria, the structural importance of the individual element within the system, the statistical dependence among deterioration failures throughout the structure and the inspection and repair policy. Let $\beta_{D S}^{T}$ denote the target reliability index associated with the deteriorated structural system and $\beta_{D E i}^{T}$ denote the target reliability index for deterioration failure of the $i^{\text {th }}$ element in the structure. Our objective is to determine $\beta_{D E i}^{T}$ so that the overall system complies with $\beta_{D S}^{T}$. This approach is motivated by the Probabilistic Model Code of the Joint Committee on Structural Safety, JCSS (2006), where target reliabilities are specified for the entire structural system based on socio-economical principles, including life safety aspects.

\section{Target reliability indices for the structural system}

In the Probabilistic Model Code of the Joint Committee on Structural Safety (JCSS 2006), target reliability indices $\beta^{T}$ for ultimate limit states are specified as a function of the consequences of component failure and the relative cost of a safety measure, see Table 1 . This differentiation reflects the fact that the target reliability indices are based on an optimization of expected life-cycle costs (Rackwitz 2000). The values in Table 1 are equally valid for new and existing structures, but the relative cost of safety measures is typically larger for the latter, leading to generally lower target reliability indexes for existing structures. 
Table 1. Tentative target reliability indices $\beta^{T}$ for ultimate limit states and one year reference period, as recommended in JCSS (2006).

\begin{tabular}{|l|l|l|l|}
\hline $\begin{array}{l}\text { Relative cost of } \\
\text { safety measure }\end{array}$ & $\begin{array}{l}\text { Minor consequences } \\
\text { of failure }\end{array}$ & $\begin{array}{l}\text { Moderate consequences } \\
\text { of failure }\end{array}$ & $\begin{array}{l}\text { Large consequences } \\
\text { of failure }\end{array}$ \\
\hline Large & $\beta^{T}=3.1\left({p_{F}}^{T} \approx 10^{-3}\right)$ & $\beta^{T}=3.3\left(p_{F}{ }^{T} \approx 5 \cdot 10^{-3}\right)$ & $\beta^{T}=3.7\left(p_{F}{ }^{T} \approx 10^{-4}\right)$ \\
\hline Normal & $\beta^{T}=3.7\left({p_{F}}^{T} \approx 10^{-4}\right)$ & $\beta^{T}=4.2\left(p_{F}{ }^{T} \approx 10^{-5}\right)$ & $\beta^{T}=4.4\left(p_{F}{ }^{T} \approx 5 \cdot 10^{-6}\right)$ \\
\hline Small & $\beta^{T}=4.2\left({p_{F}}^{T} \approx 10^{-5}\right)$ & $\beta^{T}=4.4\left({p_{F}}^{T} \approx 5 \cdot 10^{-6}\right)$ & $\beta^{T}=4.7\left(p_{F}{ }^{T} \approx 10^{-6}\right)$ \\
\hline
\end{tabular}

According to JCSS (2006), "the values given [in Table 1] relate to the structural system or in approximation to the dominant failure mode." In the absence of owner-specified reliability targets, these values can be considered as the target reliability indices associated with deterioration-induced system collapse, $\beta_{D S}^{T}$. Mitigation measures against deterioration typically are expensive, in particular for existing structures, and the target reliability indices in most cases will be as given in the upper two lines of Table 1 .

\section{System model}

To verify compliance with $\beta_{D S}^{T}$, a model for computing the probability of collapse of the deteriorating structural system, $p_{D S}$, and the corresponding reliability index $\beta_{D S}$, is needed. Deterioration in a system is deemed acceptable if $\beta_{D S}^{T} \leq \beta_{D S}$. We propose a formulation based on a simplified model of the element and system behavior. The first simplification is that, on a system level, deterioration of any element $i$ at time $t$ is modeled by a binary random process $E_{i}(t)$ with outcome space $\left\{F_{i}, \bar{F}_{i}\right\}, F_{i}$ being the event of deterioration failure of the element and a superposed bar indicating the complement. Thus, no gradual decay of the element strength is considered: At a given time $t$, the element either has its full capacity (not deteriorated) or has completely lost its capacity due to deterioration. (The appropriateness of this idealization is discussed later in the section on deterioration models.) The deterioration 
state of the system, represented by the random process $\Psi(t)$, is a function of $E_{i}(t)$, $i=1,2, \ldots, n$, where $n$ is the number of deteriorating structural elements. The outcome space of $\Psi(t)$ thus consists of $2^{n}$ disjoint states, $\psi_{i}, i=1, \ldots, 2^{n}$. The first of these states corresponds to the event of no deterioration failure in the structural system, $\psi_{1}=\left\{\bar{F}_{1} \cap \overline{F_{2}} \cap \ldots \cap \overline{F_{n}}\right\}$, and the last to the event that all elements have failed due to deterioration, $\psi_{2^{n}}=\left\{F_{1} \cap F_{2} \cap \cdots \cap F_{n}\right\}$.

A second simplification is that the deterioration state of the system is constant over a time period $\Delta t=1$ year, which is considered to be small in relation to the service life of the structure. To be on the conservative side, the system deterioration state in the period $(t-\Delta t, t]$ is set equal to the state at time $t, \Psi(t)$. The event of structural collapse in that time interval is denoted by $C(t)$. The probability of this event conditioned on the deterioration state of the system at time $t, \operatorname{Pr}[C(t) \mid \Psi(t)]$, can be computed by performing reliability analyses of the structure with the elements damaged according to $\Psi(t)$, i.e., all elements that are failed due to deterioration are removed in the structural model employed in the reliability analysis. However, for real structures it is not feasible to evaluate all $2^{n}$ values of $\operatorname{Pr}[C(t) \mid \Psi(t)]$, as this would require an enormously large number of system reliability analyses $\left(2^{n}\right.$ being the size of the outcome space of $\Psi(t))$. To circumvent this problem, later in this paper we propose to compute $\operatorname{Pr}[C(t) \mid \Psi(t)]$ for an approximately equivalent idealized system, which is constructed based on a set of indicators of the real structural system.

The probability of structural collapse in the reference period $(t-\Delta t, t]$ is given by the total probability theorem as

$$
p_{D S}(t)=\operatorname{Pr}[C(t)]=\sum_{i=1}^{2^{n}} \operatorname{Pr}\left[C(t) \mid \Psi(t)=\psi_{i}\right] \operatorname{Pr}\left[\Psi(t)=\psi_{i}\right]
$$


The associated reliability index is $\beta_{D S}(t)=-\Phi^{-1}\left[p_{D S}(t)\right]$, where $\Phi^{-1}$ is the inverse of the standard normal cumulative distribution function. $\operatorname{Pr}\left[\Psi(t)=\psi_{i}\right]$ in Equation (1) is obtained as a function of probabilities of the element deterioration failure events $E_{i}(t)=F_{i}$, accounting for the statistical dependence among these events. Because we can set $\operatorname{Pr}\left[E_{i}(t)=F_{i}\right]=\Phi\left(-\beta_{D E i}^{T}\right)$ for an element designed at the limit of the acceptance criterion, Equation (1) establishes the connection between the system criterion $\beta_{D S}^{T} \leq \beta_{D S}$ and the target deterioration reliability indices of the individual elements, $\beta_{D E i}^{T}, i=1,2, \ldots, n$. Obviously, the single condition $\beta_{D S}^{T} \leq \beta_{D S}$ is not sufficient to determine the $n$ individual quantities $\beta_{D E i}^{T}$ and additional rules are required. Such rules are proposed in this paper, based on the same equivalent idealized system as introduced for computing $\operatorname{Pr}\left[C(t) \mid \Psi(t)=\psi_{i}\right]$.

\section{Modeling deterioration failure events}

Deterioration is modeled at the level of structural elements, e.g., structural members, welded joints, area segments of a continuous surface. The event of deterioration failure of element $i$ at time $t$ is represented by a limit-state function $g_{i}(\mathbf{X}, t)$, with $\mathbf{X}$ being a vector of random variables that describe the deterioration model, so that $\left\{E_{i}(t)=F_{i}\right\}=\left\{g_{i}(\mathbf{X}, t) \leq 0\right\}$. The corresponding failure probability, $\operatorname{Pr}\left[E_{i}(t)=F_{i}\right]$, can be computed by the methods of structural reliability analysis. An example deterioration limit state model is

$$
g(\mathbf{X}, t)=D-A t^{B}
$$

where $t$ is the time since installation or repair of the element, $D$ is the damage limit and $A$ and $B$ are parameters describing the deterioration process. For $B=1$, this corresponds to most applied corrosion models as well as to the Palmgren-Miner fatigue model with a stationary 
stress process; for $B=0.5$, the model is representative of diffusion-controlled deterioration, and for $B=2$ the model approximates concrete deterioration due to sulfate attack.

Deterioration in an element occurs gradually with time and representing the capacity of such an element by the two-state random process $E_{i}(t)$ is a strong simplification. Therefore, care is required in defining the failure criterion in the deterioration limit state function, such as $D$ in Equation (2). If the failure event is defined so that the capacity of the element is significantly reduced before the limit state is reached, the binary model can be unconservative. On the other hand, if the failure event is defined so that the element is considered failed after a small loss of capacity, the model will give conservative results for the system. In general, the assumed binary model would be most appropriate when the deterioration initiates and failure occurs within the same time interval $(t-\Delta t, t]$.

For fatigue deterioration, limit states provided in codes generally correspond to defect initiation or the event of a through-thickness crack and not to loss of capacity; the remaining capacity of the element or joint at the limit state may be close to its capacity in the undamaged state. Therefore, the proposed model is conservative for fatigue limit states; however, the degree of conservatism can vary. For some structural details, fatigue can lead to unstable crack growth and complete loss of capacity shortly after reaching the limit state and the model is accurate. On the other hand, in many structural configurations loads redistribute once a loss of stiffness occurs and crack growth slows down after the limit state is reached; the model is conservative in this case. Despite its potential conservatism, we believe the proposed binary model is justified for modeling high-cycle fatigue failures in engineering practice. For low-cycle fatigue, however, the model can be non-conservative. Damaging stress cycles due to low-cycle fatigue usually occur during extreme events, and it is more probable that deterioration failures and 
structural collapse occur during the same load event. By not accounting for this likely concurrence, the model might underestimate the probability of collapse.

The binary model is suitable for other deterioration processes that lead to rapid reduction of capacity after an initiation period. These include various forms of stress corrosion cracking and deterioration processes that are controlled by a protection system. In the latter case, the deterioration failure event should be defined (conservatively) as the failure of the protection system.

For other deterioration mechanisms that lead to slow reduction of the element capacity, such as uniform corrosion or distributed pitting corrosion on steel surfaces and on reinforcement of RC structures, the binary model is less appropriate. It might still be applied if the failure criterion is selected conservatively, e.g., by defining the allowable corrosion loss in ship structures as the damage limit in Equation (2) or by defining the failure of the reinforcement as corrosioninduced loss of bond. Depending on the application, the results obtained with the model presented in this paper can be overly conservative and approaches based on structure-specific system reliability analyses might become necessary. However, it is noted that for deterioration of RC structures, serviceability limit states are often found to be determining the required level of deterioration reliability (Stewart and Val 2003). In this case, the present approach can still be used to check whether the reliability levels implied by the serviceability criteria are complying with the system safety criterion.

\section{Modeling statistical dependence among deterioration failure events}

The deterioration failure events of elements in a structural system are generally statistically dependent due to common uncertain influencing factors, such as environmental conditions and 
material characteristics. Statistical dependence among element deterioration failures can be expressed through the correlation coefficients among the corresponding limit state functions. As an example, consider the deterioration limit state in Equation (2). This can be reformulated into the equivalent form

$g(\mathbf{X}, t)=\ln D-\ln A-B \ln t$

If both $D$ and $A$ are modeled by a Lognormal distribution and $B$ is modeled by a Normal distribution, assuming independence of the three variables, the reliability index at time $t$ without inspection becomes

$\beta_{D E i}(t)=\frac{\lambda_{D}-\lambda_{A}-\mu_{B} \ln t}{\sqrt{\zeta_{D}^{2}+\zeta_{A}^{2}+\left(\sigma_{B} \ln t\right)^{2}}}$

with $\lambda_{D}, \lambda_{A}$ and $\mu_{B}$ being the means of $\ln \Delta, \ln A$ and $B$, and $\zeta_{\Delta}, \zeta_{A}$ and $\sigma_{B}$ being the corresponding standard deviations, respectively. As an example, assume the statistical dependence between the deterioration failures of two elements $i$ and $j$ arises due to correlation between the corresponding variables $\ln D_{i}$ and $\ln D_{j}$ and between $\ln A_{i}$ and $\ln A_{j}$, denoted $\rho_{\ln A}$ and $\rho_{\ln \Delta}$, respectively, while variable $B$ remains statistically independent from element to element. Assuming identical marginal probability distributions of these variables for the two elements, the correlation coefficient between the corresponding pairs of limit states functions is

$\rho_{M}(t)=\frac{\rho_{\ln \Delta} \zeta_{\Delta}^{2}+\rho_{\ln A} \zeta_{A}^{2}}{\zeta_{\Delta}^{2}+\zeta_{A}^{2}+\left(\sigma_{B} \ln t\right)^{2}}$ 
For the special case considered here, with the limit-state functions being jointly normally distributed, the pair-wise correlation coefficients $\rho_{M}(t)$ together with $\beta_{D E i}(t)$ fully describe the probability mass function (PMF) of $\Psi(t)$, i.e., the probabilities of all possible combinations of element deterioration failures in the system. In the more general case, when the deterioration limit state function is not linear and the random variables are not normal or lognormal, $\rho_{M}(t)$ can be taken as the correlation coefficient between the linearized limit states obtained from a FORM solution of a parallel system with two elements (Ditlevsen and Madsen 1996).

\section{Investigation of earlier models for developing deterioration acceptance criteria in redundant structural systems}

In principle, to establish the element acceptance criterion $\beta_{D E i}^{T}$ as a function of the system acceptance criterion $\beta_{D S}^{T}$, it is required to solve Equation (1). Because of the difficulty in computing $\operatorname{Pr}[C(t) \mid \Psi(t)]$ for all outcomes of $\Psi(t)$, existing approaches (HSE 2002, Ronalds et al. 2003, Moan 2005, Straub and Faber 2005a) employ an approximate version of Equation (1). As an example, HSE (2002) utilizes the following approximation:

$$
p_{D S}(t) \approx \operatorname{Pr}\left[C(t) \mid \Psi(t)=\psi_{1}\right]+\sum_{i=1}^{n} \operatorname{Pr}\left[C(t) \mid \Psi(t)=\left(\overline{F_{1}} \cap \ldots \cap \overline{F_{i-1}} \cap F_{i} \cap \overline{F_{i+1}} \cap \ldots \cap \overline{F_{n}}\right)\right] \operatorname{Pr}\left[E_{i}(t)=F_{i}\right]
$$

Here, the influence of individual deterioration failures $F_{i}$ is appraised through the probability of system failure with element $i$ removed and all other elements intact: $\operatorname{Pr}\left[C(t) \mid \Psi(t)=\left(\overline{F_{1}} \cap \ldots \cap \overline{F_{i-1}} \cap F_{i} \cap \overline{F_{i+1}} \cap \ldots \cap \overline{F_{n}}\right)\right]$. This conditional probability has often been used as an indicator for redundancy of the structure with respect to failure of element $i$ (Lotsberg and Kirkemo 1989, Gharaibeh et al. 2002). The approach based on Eq. (6) 
requires only one additional reliability analysis per element, i.e., $n$ analyses instead of $2^{n}$, which makes it practically feasible. By comparing Equations (1) and (6), it can be seen that the two formulations are identical if the element deterioration failure events are mutually exclusive and if the probability of collapse of the intact structure is zero, $\operatorname{Pr}\left[C(t) \mid \Psi(t)=\psi_{1}\right]=0$. As discussed in Straub and Faber (2005a), and as demonstrated by a numerical example later in this paper, the approximation is reasonable when the individual structural elements have high deterioration reliability $\left(\beta_{D E i}(t) \geq 3.5\right)$, when the number of structural elements is small and when deterioration failure events are uncorrelated. In such a case, the probability of the joint occurrence of two or more deterioration failures becomes negligible. (If all elements have the same failure probability $\operatorname{Pr}\left[E_{i}(t)=F_{i}\right]=p$, the probability of more than one statistically independent failure event among $n$ elements is $1-(1-p)^{n}-n p(1-p)^{n-1} \cong n(n-1) p^{2} / 2$, which is much smaller than $p$ when $p$ is small and $n$ is of order smaller than $1 / \sqrt{p}$.) Unfortunately, these conditions are not generally fulfilled for real structures.

Motivated by the approximation in Equation (6), we define the Single-Element Importance (SEI) measure for element $i$ as

$$
S E I_{i}=\operatorname{Pr}\left[C(t) \mid \Psi(t)=\left(\overline{F_{1}} \cap \ldots \cap \overline{F_{1-1}} \cap F_{i} \cap \overline{F_{1+1}} \cap \ldots \cap \overline{F_{n}}\right)\right]-\operatorname{Pr}\left[C(t) \mid \Psi(t)=\psi_{1}\right]
$$

As can be seen, $S E I_{i}$ is the difference in the failure probability of the system with all elements intact (not deteriorated) and the system where only element $i$ has failed due to deterioration.

In addition to Equation (6), further conditions are required to establish the element acceptance criteria. It has been suggested, explicitly in (Straub and Faber 2005a) and implicitly in (Ronalds et al. 2003, Moan 2005, HSE 2002), to determine the $\beta_{D E i}^{T}$ such that all summation terms in 
Equation (6) are equal, i.e., all elements contribute equally to the probability of system failure associated with deterioration. The target reliability indices for all elements are then obtained as:

$$
\beta_{D E i}^{T}=-\Phi^{-1}\left\{\frac{1}{n} \frac{\Phi\left(-\beta_{D S}^{T}\right)-\operatorname{Pr}\left[C(t) \mid \Psi(t)=\psi_{1}\right]}{S E I_{i}+\operatorname{Pr}\left[C(t) \mid \Psi(t)=\psi_{1}\right]}\right\}
$$

Both Equations (6) and (8) neglect the contribution of joint deterioration failure events of two or more elements. To examine this effect, in the following an idealized system, for which $\operatorname{Pr}[C(t) \mid \Psi(t)]$ is easily computable, is investigated.

To simplify the notation, hereafter $\beta_{D E i}(t)$ is written as $\beta_{D E i}$, because the structure is verified under the assumption that the element deterioration reliability is at its limit, i.e. $\beta_{D E i}(t)=\beta_{D E i}^{T}$, which does not depend on time. In addition, the random variables $C(t)$ and $\Psi(t)$ are written as $C$ and $\Psi$, since the probability $\operatorname{Pr}[C \mid \Psi]$ does not change with time under the common assumption that the distribution of the annual maximum load is constant with time and $\operatorname{Pr}(\Psi)$ does not change with time if the $\beta_{D E i}$ are constant with time.

\section{The SEI for a Daniels system}

Consider the Daniels system (Daniels 1945) shown in Figure 1. The elements of the system have independent and identically distributed (iid) capacities, i.e. they are exchangeable in the statistical sense. In Gollwitzer and Rackwitz (1990), the characteristics of this system are examined for a variety of element behaviors. This idealized system is well suited for representing the load-sharing phenomenon present in structural systems, with the two cases (a) and (b) in Figure 1 representing the extremes of true material behavior. Note that the distinction between the brittle and ductile failure modes relates to element failures due to overloading of 
the structure. Deterioration, on the other hand, affects the capacities of the elements. In the simplified model considered here, the deterioration failure of an element is tantamount to reduction of its capacity to zero. The deterioration state of the system essentially dictates the number of elements that are available to resist the applied load through either a ductile or brittle behavior.

For the idealized system, computation of the $S E I_{i}$ according to Equation (7) is straightforward. The two needed terms are

$$
\begin{aligned}
& \operatorname{Pr}\left(C \mid \Psi=\left\{\overline{F_{1}} \cap \ldots \cap \overline{F_{1-1}} \cap F_{i} \cap \overline{F_{1+1}} \cap \ldots \cap \overline{F_{n}}\right\}\right)=\operatorname{Pr}\left(C \mid N_{F}=1\right) \\
& \operatorname{Pr}\left(C \mid \Psi=\psi_{1}\right)=\operatorname{Pr}\left(C \mid N_{F}=0\right)
\end{aligned}
$$

where $N_{F}$ is the number of elements failed due to deterioration.
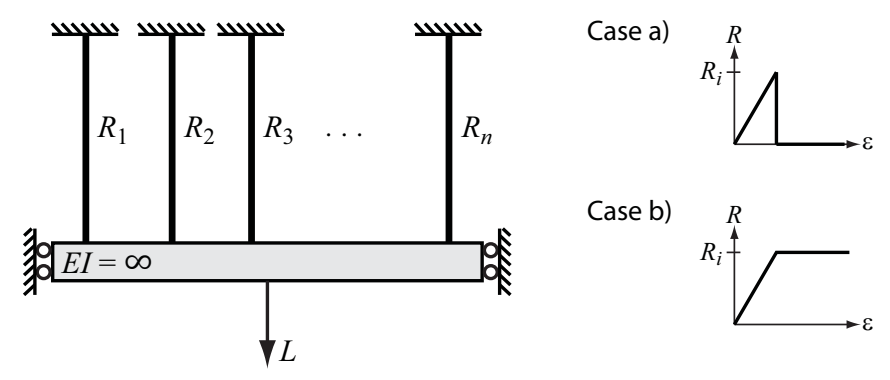

Figure 1. Idealized structural system under external load. Case a) brittle element behavior (original Daniels system), b) ductile element behavior.

To evaluate Equations (9) and (10), the conditional failure probability $\operatorname{Pr}\left(C \mid N_{F}=j\right)$ is required. For given probability distributions of the element capacities $R_{i}$ and the load $L$, this 
is readily obtained for the above system. In accordance with the definition of $C, L$ is the maximum load in the period $(t-\Delta t, t]$. For case a), the required conditional probability is calculated as

$$
\operatorname{Pr}\left(C \mid N_{F}=j\right)=\int_{L} \operatorname{Pr}[C \mid l,(n-j)] f_{L}(l) d l
$$

where the probability of system failure for given load $l$ and number of surviving elements $(n-j), \operatorname{Pr}[C \mid l,(n-j)]$, is computed according to the solution provided in Daniels (1945). For case $b$ ), the solution is given by

$$
\operatorname{Pr}\left(C \mid N_{F}=j\right)=\operatorname{Pr}\left(\sum_{i=1}^{n-j} R_{i}-L \leq 0\right)
$$

which is easily computed using structural reliability methods.

Because of exchangeability of its elements, Equation (1) for the Daniels system simplifies to

$$
p_{D S}=\sum_{i=0}^{n} \operatorname{Pr}\left(C \mid N_{F}=j\right) \operatorname{Pr}\left(N_{F}=j\right)
$$

The probability that $j$ elements have failed due to deterioration, $\operatorname{Pr}\left[N_{F}=j\right]$, is a function of the element deterioration reliability indices $\beta_{D E i}$ and the correlation coefficients $\rho_{M}$ between their limit states. We assume $\beta_{D E i}$ is the same for all elements and $\rho_{M}$ is the same for all pairs of elements. The probability of $j$ deterioration failures among $N$ elements then is 
$\operatorname{Pr}\left[N_{F}=j\right]=\left(\begin{array}{c}n \\ n-j\end{array}\right) \int_{-\infty}^{\infty} \varphi(u) p(u)^{n-j} p(u)^{j} d u$

with $p(u)=\Phi\left[\frac{\beta_{D E i}-u \sqrt{\rho_{M}}}{\sqrt{1-\rho_{M}}}\right]$

where $\varphi()$ is the standard Normal probability density function. This equation is based on a binomial model with uncertain parameter $p$, which accounts for the statistical dependence among the Bernoulli trials according to the correlation coefficient $\rho_{M}$.

\section{Numerical investigations}

With the Daniels system as an example of a structural system, we can now investigate the effect of the approximation made in existing approaches for determining the deterioration target reliability index. This is done by comparing the true deterioration reliability of the Daniels system with the one computed according to Equation (6). For this purpose, the load, $L$, is modeled by a lognormal distribution with coefficient of variation (c.o.v.) $\delta_{L}=0.25$ and the capacities of the elements, $R_{i}$, are modeled by independent and identical normal distributions with c.o.v. $\delta_{R}=0.15$. The ratio of the mean values of $n R_{i}$ and $L$, which can be considered as the mean safety factor for system overload failures, is determined such that the system in its undamaged state (without deterioration failures) has reliability index $\beta_{\overline{D S}}=-\Phi^{-1}\left\{\operatorname{Pr}\left[C \mid \Psi=\psi_{1}\right]\right\}=4.4$. (This value has reference period $\Delta t=1 \mathrm{yr}$, but is not dependent on time $t$.) For a system with $n=20$ elements, this gives $n \mu_{R_{i}} / \mu_{L}=3.67$ for the brittle material behavior and $n \mu_{R_{i}} / \mu_{L}=2.90$ for the ductile behavior. For this system, $\operatorname{Pr}\left(C \mid N_{F}=j\right)$ is illustrated in Figure 2 as a function of $j$ for the two material models as computed by use of Equation (14). It is observed that the criticality of deterioration failures is almost identical for the two material behaviors. (It is reminded that the difference in material 
behaviors relates only to overload failures. Deterioration failures for both material behaviors are modeled as brittle, i.e., without remaining load capacity.) In the remainder of this section, only the system with ductile elements is considered.

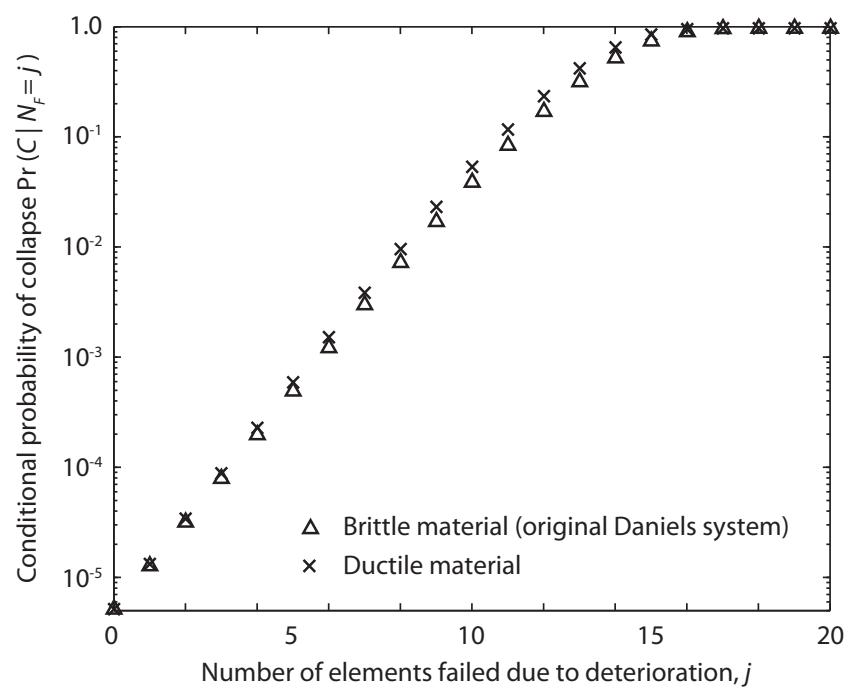

Figure 2. Failure probability of Daniels system as a function of the number of elements failed due to deterioration.

To appraise the effect of the approximation introduced in previous approaches to determining the deterioration acceptance criteria, we compute the system reliability associated with deterioration failures, $\beta_{D S}=-\Phi^{-1}\left(p_{D S}\right)$, according to Equation (6) and Equation (13). Equation (6) represents the approximation used in previous approaches and is based on the $S E I_{i}$, which here is the same for all elements and is computed as $S E I_{i}=\operatorname{Pr}\left(C \mid N_{F}=1\right)-\operatorname{Pr}\left(C \mid N_{F}=0\right)$. Equation (13) gives the exact value of $\beta_{D S}$ for the Daniels system and is used as a reference. In Figure $3, \beta_{D S}$ is shown as a function of the number of elements, $n$, the deterioration reliability index of the individual elements, $\beta_{D E_{i}}$, and the pair-wise correlation coefficient among the deterioration safety margins, $\rho_{M}$. 

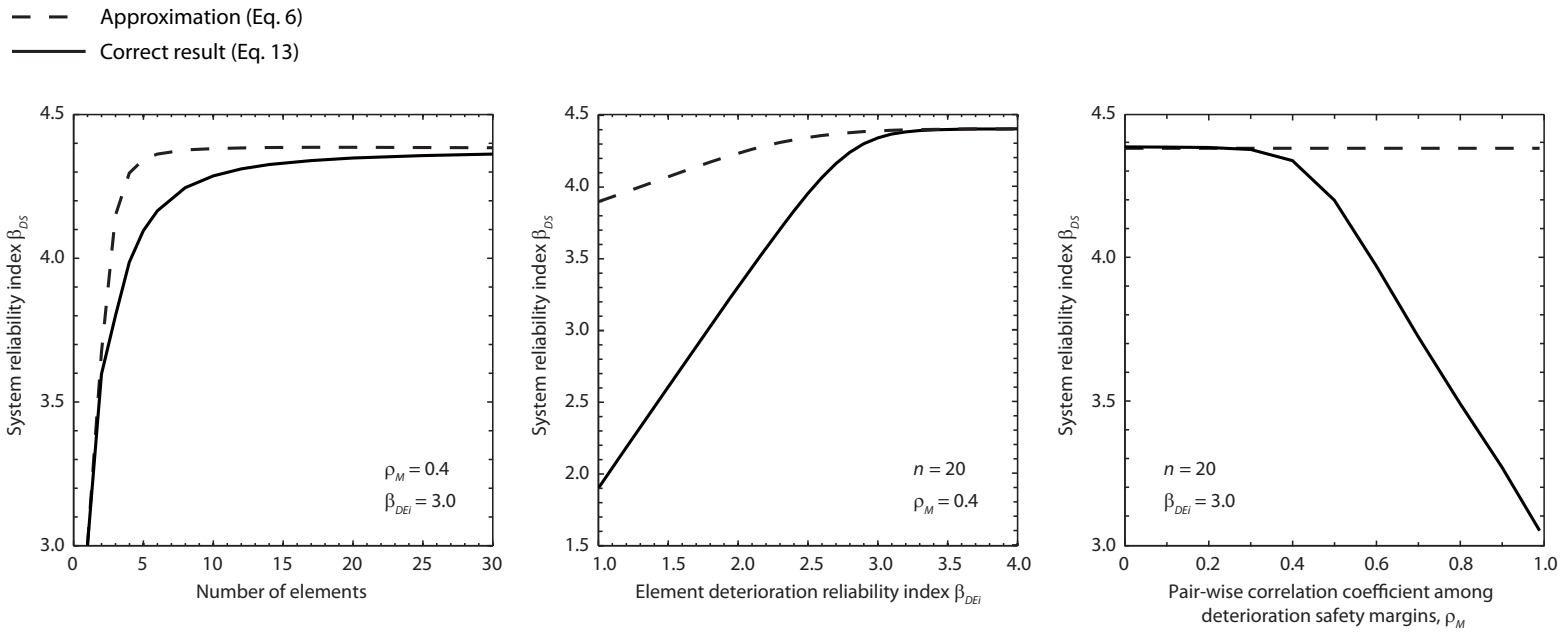

Figure 3. System deterioration reliability index as a function of number of elements (left chart), element deterioration reliability index (middle chart), and correlation among deterioration limit states (right chart).

The results in Figure 3 clearly demonstrate that the approximation made in previous approaches to determining deterioration acceptance criteria overestimates the reliability of the investigated system, and the same tendency is expected for every redundant structural system. This effect is relatively constant with the number of elements in the Daniels system, $n$, except when $n$ is close to one, representing systems with limited or no redundancy. Furthermore, as mentioned earlier, the approximation is close to the correct result when the deterioration reliability index of the individual elements is large and when the statistical dependence among deterioration failure events is low $\left(\rho_{M} \leq 0.3\right)$. In these cases, the probability of joint occurrence of several element deterioration failures is negligible. However, for most real structural systems, these assumptions do not hold, and an improved approximation to the actual system deterioration reliability $\beta_{D S}$ is required. Such an approximation is presented and investigated in the remainder of this paper. 


\section{Acceptance criteria for deteriorating structural elements in general redundant systems}

\section{Equivalent structural systems}

Our aim here is to set a target reliability index $\beta_{D E i}^{T}$ for each deteriorating element of a structural system so that the system reliability index considering deterioration, $\beta_{D S}$, is no less than a specified target reliability index $\beta_{D S}^{T}$. Obviously $\beta_{D E i}^{T}$ may need to be different for different elements, depending on the relative structural importance of each element. The relationship between element and system reliability indices, however, is an intricate one, governed by the nature of load-sharing between the elements, the configuration of the system and, in particular, the distribution of deteriorating elements within the structure. It is impractical to use an exact representation of the system (e.g., as a series system of parallel subsystems, Hohenbichler and Rackwitz 1982) to establish this relationship. Instead, here we make use of an idealized "equivalent" representation of the system to determine the required relationship. It is desirable to choose an idealized system with exchangeable (statistically independent and identically distributed) elements, because this property facilitates computation of the relation between $\beta_{D E i}^{T}$ and $\beta_{D S}$, as earlier demonstrated for the Daniels system.. However, the elements in the real structure have varying importance and cannot be represented as exchangeable elements within a single idealized system. Therefore, a different idealized system is defined for each deteriorating element in the real structure.

For the idealized system to provide an accurate representation, it must be calibrated to the reliability characteristics of the real element and the real structure. Hence, for each element, the corresponding idealized system is defined so that it correctly represents the reliability of the intact structure and the reliability of the structure with the element removed. The difference between these two reliability measures, which is equal to the SEI of the element, in a sense 
reflects the redundancy of the real system with respect to the selected element. Additionally, the idealized system should reflect the total number of deteriorating elements in the real structure, $n$. This is because, for given reliability of the intact structure and its redundancy with respect to the selected element, a larger $n$ implies a higher number of failure modes and consequently lower system reliability. To assure satisfaction of the overall system reliability requirements, the target reliability index for the selected element must account for $n$.

For each element $i$ in the real structure, the proposed equivalent idealized system consists of a set of $k$ Daniels subsystems in series, each having $n_{i}$ elements with statistically independent and identically distributed capacities. $n_{i}$ is selected so that it represents the redundancy of the real structural system with respect to deterioration failure of element $i$; when this redundancy is large, equivalent Daniels subsystems with larger number of elements are used, wherein failure of one element has a smaller effect. Since $n_{i}$ is determined purely based on the redundancy of the system with respect to element $i$, it does not reflect the total number of elements in the real system. For this reason, $k$ subsystems are considered in series, where $k$ is selected to appropriately represent the total number of elements in the real structure $n$. A larger value of $n$ for constant $n_{i}$ implies a larger value of $k$. The numerical determination of $n_{i}$ and $k$ is described later.

The deterioration failure events of the elements within each Daniels system with $n_{i}$ elements are characterized by the common target reliability index $\beta_{D E i}^{T}$ and the common correlation coefficient $\rho_{M}$, which represents the dependence of the deterioration failure of element $i$ on those of other elements, e.g. computed according to Eq. (5). Deterioration failure events in different Daniels subsystems are assumed to be statistically independent. The loads acting on the $k$ subsystems are statistically independent and identically distributed. Due to this latter 
assumption, which is necessary to maintain exchangeability of the elements, the system cannot be interpreted as a single structural system. Instead, it is a logical system, which fails if any of its $k$ Daniels subsystems fails. The idealized system is illustrated in Figure 4.

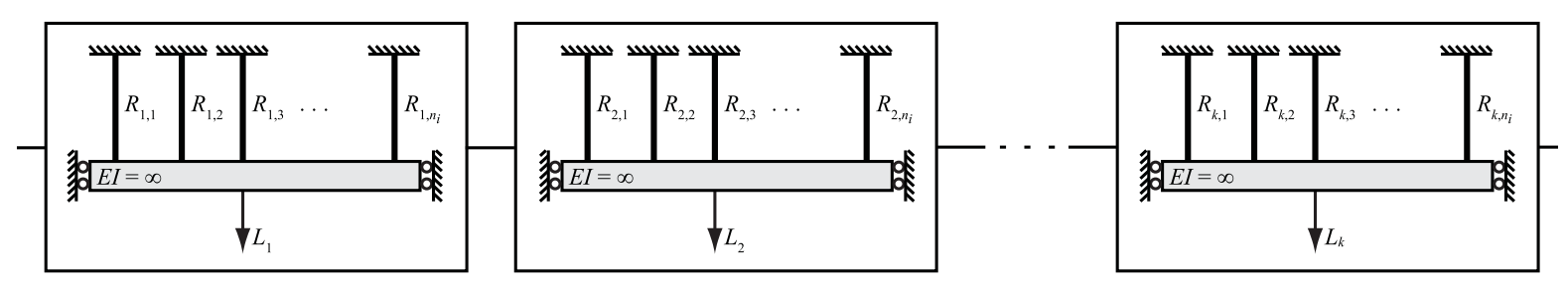

Figure 4. The equivalent system for element $i$. In their undeteriorated state, all $n_{i} \cdot k$ elements have independent and identically distributed capacities $R_{i, j}$ relative to overload failure. System failure occurs if any of the $k$ subsystems fails.

The distributions of the loads $L_{j}$ and the element capacities $R_{j, i}$ must be selected so as to represent the characteristics of the dominant load case, and the parameters are selected so that the idealized system in its intact state has the same reliability as the real structure without deterioration failures. As an example, for an offshore structure in a hurricane-prone area, typical values of the c.o.v. are $\delta_{L}=0.35$ and $\delta_{R_{i}}=0.15$ (Stahl et al. 2000). These values are utilized in the numerical examples in this paper, and it is assumed that $L_{j}$ is modeled by a Lognormal distribution and $R_{j, i}$ by a Normal distribution. The ratio between the mean values of $L_{j}$ and $n_{i} R_{j, i}$ is determined by matching the reliabilities of the real and idealized systems in their intact (not deteriorated) states. Specifically, the ratio $n_{i} \mu_{R_{i}} / \mu_{L}$ is determined iteratively from the condition

$\operatorname{Pr}\left(C_{i} \mid N_{F}=0\right)=1-\left[\Phi^{-1}\left(\beta_{\overline{D S}}\right)\right]^{\frac{1}{k}}$ 
where $\beta_{\bar{D} S}$ is the reliability index of the real structure in its intact state, $C_{i}$ is the event of failure of a Daniels system with $n_{i}$ elements and $\operatorname{Pr}\left(C_{i} \mid N_{F}=0\right)$ is computed according to Equation (11) or (12).

$n_{i}$, the number of elements in each Daniels system, represents the redundancy of the real structural system with respect to deterioration failure of element $i$. Specifically, $n_{i}$ is selected as the number of elements of the Daniels system for which the (exchangeable) elements have the same SEI as element $i$ in the real structure. The SEI of the elements in the equivalent system, denoted by $S E I_{i}^{\prime}$, is obtained as

$$
S E I_{i}^{\prime}=1-\left[1-\operatorname{Pr}\left(C \mid N_{F}=0\right)\right]^{k-1}\left[1-\operatorname{Pr}\left(C \mid N_{F}=1\right)\right]-\Phi\left(-\beta_{\overline{D S}}\right)
$$

Here, $\operatorname{Pr}\left(C \mid N_{F}=j\right)$ is the probability of failure of a Daniels system with $n_{i}$ elements, of which $j$ elements have failed due to deterioration, and is given by Equations (11) and (12). Since $n_{i}$ is an integer variable, the $S E I_{i}$ computed for element $i$ of the real structure cannot be exactly matched. Instead, the two integer values of $n_{i}$ that give $S E I_{i}^{\prime}$ values closest to $S E I_{i}$ are determined and the analysis is carried out for the two systems.

Since $n_{i}$ is not a direct function of the number of deteriorating elements in the real structure $n$, the effect of $n$ on the system reliability is accounted for by $k$, the number of Daniels systems in series. For given values $n_{i}, i=1, \ldots, n, k$ can be determined as the sum of the contributions of the elements in their respective equivalent systems, which can be stated as

$$
k=\sum_{i=1}^{n} \frac{1}{n_{i}}
$$


Alternatively, $k$ can be determined from the condition that the mean number of elements in the equivalent subsystems should be equal to the true number of deteriorating elements. It then follows that

$$
k=\frac{n^{2}}{\sum_{i=1}^{n} n_{i}}
$$

Hereafter, we employ Equation (17), but we note that Equation (18) gives similar results and both formulations give exact results in the extremes: for a series system with $n$ elements where $n_{i}=1$ for all elements, both equations correctly give $k=n$, and for a parallel system with $n$ elements where $n_{i}=n$, they correctly give $k=1$.

So far we have described how the parameters defining the equivalent systems, i.e., $k$, $n_{i}, i=1, \ldots, n$ and the ratio between the mean values of $L_{j}$ and $n_{i} R_{j, i}$, are obtained separately, assuming that the other parameters are given. To determine all parameters jointly, an iterative procedure is utilized. An initial guess of $k$ is made, and the remaining parameters are determined for the given $k$. With the resulting values of $n_{i}, i=1, \ldots, n$, a new value of $k$ is computed and the process is repeated until convergence in $k$ is achieved. Figure 5 summarizes the procedure for determining the parameters of the equivalent systems. The computational effort for this procedure is reasonable and not critical for practical implementations (in the order of seconds on a standard Pentium II PC for an implementation in Matlab). 


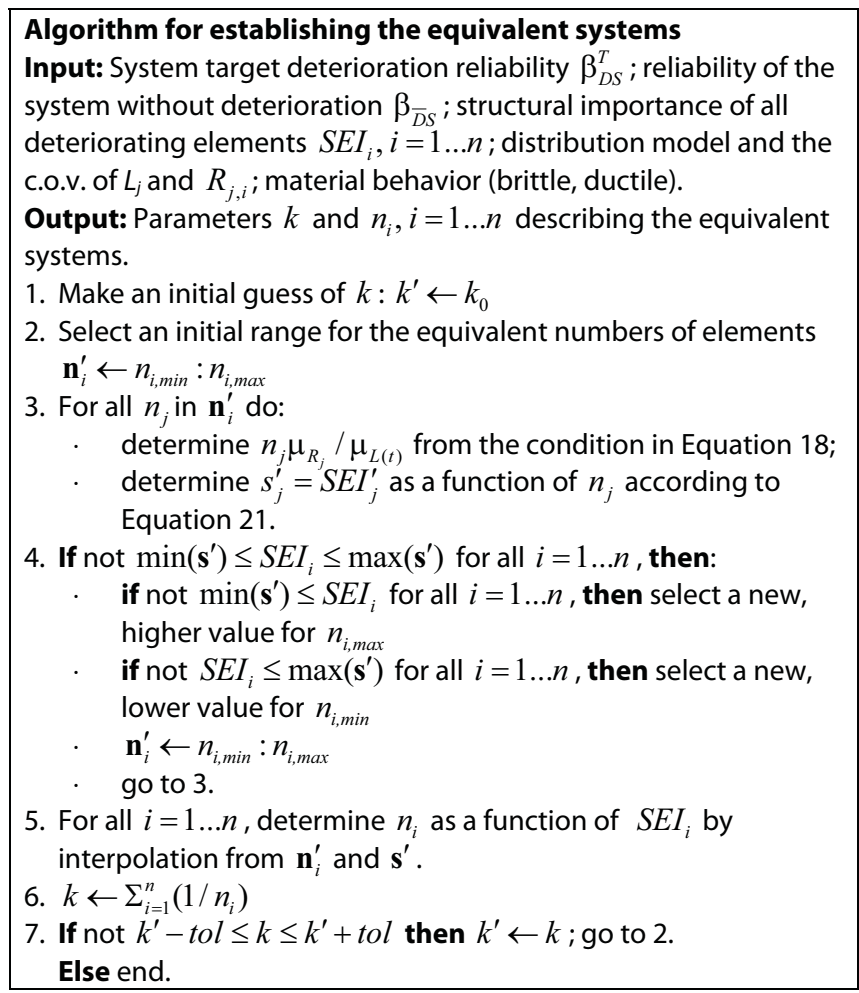

Figure 5. Algorithm for establishing the equivalent systems.

\section{Determination of the element acceptance criterion from the equivalent system}

Once the equivalent system for element $i$ is established, this system is utilized to determine the element deterioration acceptance criterion $\beta_{D E i}^{T}$. The equivalent system has exchangeable elements, so all its elements have the same deterioration reliability index. The value of $\beta_{D E i}^{T}$ is determined from the condition $\beta_{D S}=\beta_{D S}^{T}$, where $\beta_{D S}$ is the reliability index associated with deterioration failures in the equivalent system.

The probability of failure of the equivalent system is

$$
p_{D S}=\operatorname{Pr}(C)=1-\left[1-\sum_{j=0}^{n_{i}} \operatorname{Pr}\left(C \mid N_{F}=j\right) \operatorname{Pr}\left(N_{F}=j\right)\right]^{k}
$$


$\operatorname{Pr}\left(C \mid N_{F}=j\right)$ is given by Equations (11) and (12), and $\operatorname{Pr}\left(N_{F}=j\right)$ is obtained from Equation (14) as a function of $\beta_{D E i}^{T}$ and $\rho_{M}$. Finally, $\beta_{D E i}^{T}$ is obtained by finding the value that fulfills $\beta_{D S}^{T}=-\Phi^{-1}\left(p_{D S}\right)$, with $p_{D S}$ according to Equation (19).

\section{Validation}

To validate the proposed model, we apply it to the simple 2-D frame structure shown in Figure 6. This structure is chosen because, despite its small number of elements, it captures some of the characteristics of real structures. In particular, the structure exhibits redundancy with respect to individual deterioration failures. The deterioration target reliability indices of the structural elements are determined according to the proposed model. For validation, the deterioration reliability of the system designed according to these target values is then determined according to Equation (1), and is compared with the system deterioration target reliability index. This comparison requires computing the reliability index of the system for all $2^{n}$ combinations of system deterioration states.

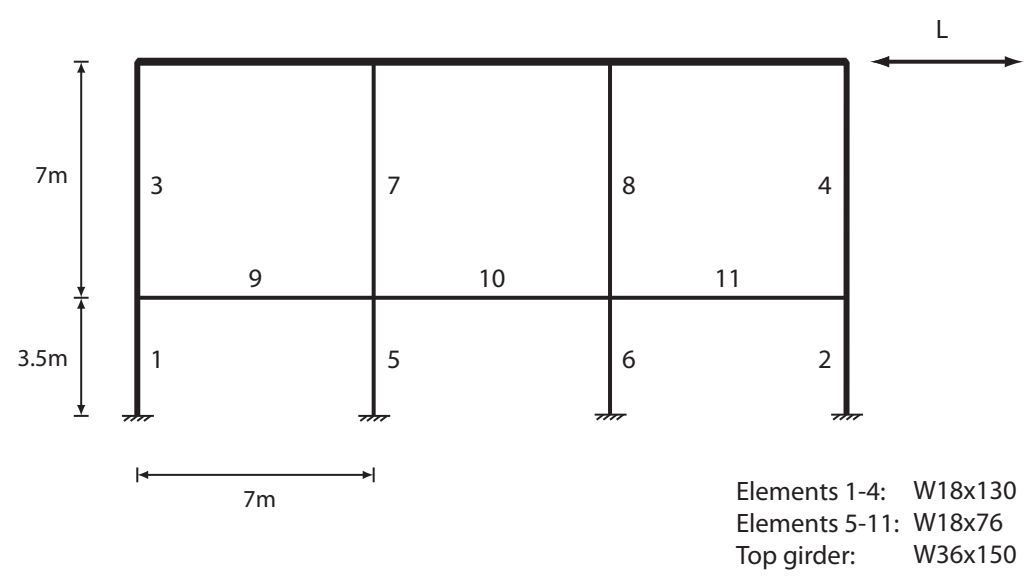

Figure 6. Structural system for model validation. 
The considered structure is subjected to a random horizontal load, whose annual maximum $L$ has the Gumbel distribution with mean $\mu_{L}=351 \mathrm{kN}$ and c.o.v. $\delta_{L}=0.35$. The material and geometrical properties of the structural elements are modeled deterministically. The capacity of the structure with respect to $L$ is evaluated using non-linear FE (pushover) analysis. For the intact structure, this capacity is assessed as $1461 \mathrm{kN}$, which implies an annual reliability index $\beta_{\overline{D S}}=4.4$. It is assumed that deterioration can occur in structural elements $1-11$, but not in the top girders. Therefore, there are $2^{11}=2048$ possible combinations of system deterioration states $\Psi$ and this number of pushover analyses are performed to evaluate $\operatorname{Pr}\left[C \mid \Psi=\psi_{i}\right]$ for all $i$.

Table 2 shows the resulting $S E I_{i}$ and corresponding $n_{i}$ values for the 11 elements, together with the target reliability indices $\beta_{D E i}^{T}$ for different cases of $\beta_{D S}^{T}$ and $\rho_{M}$, assuming ductile material behaviour. The parameter $k$, which describes the number of equivalent Daniels systems, is computed as $k=2.3$ by Equation (17). (A non-integer value of $k$ has no physical meaning, but mathematically there is no difficulty in using such a value. The results obtained with a value of $k=2.3$ lie between results obtained with $k=2$ and $k=3$.)

Table 2. Resulting deterioration target reliability indices $\beta_{D E i}^{T}$ for the validation structure.

\begin{tabular}{|c|c|c|c|c|c|c|c|c|}
\hline \multirow[t]{3}{*}{ Elements $i$} & \multirow{3}{*}{$\begin{array}{l}S E I_{i} \\
{\left[\times 10^{-3}\right]}\end{array}$} & \multirow[t]{3}{*}{$n_{i}$} & \multirow{2}{*}{\multicolumn{3}{|c|}{$\frac{\text { Target reliability index }}{\beta_{D S}^{T}=3.7} \beta_{D E i}^{T}$}} & \multirow{3}{*}{$\begin{array}{l}\beta_{D S}^{T}=4.2 \\
\rho_{M}=0.0\end{array}$} & \multirow[b]{3}{*}{$\rho_{M}=0.3$} & \multirow[b]{3}{*}{$\rho_{M}=0.6$} \\
\hline & & & & & & & & \\
\hline & & & $\overline{\rho_{M}}=0.0$ & $\rho_{M}=0.3$ & $\rho_{M}=0.6$ & & & \\
\hline 1,2 & 0.27 & 3.5 & 2.20 & 2.60 & 3.10 & 2.80 & 3.10 & 3.60 \\
\hline 3,4 & 0.69 & 2.9 & 2.40 & 2.75 & 3.15 & 3.00 & 3.25 & 3.65 \\
\hline 5,6 & 0.017 & 7.7 & 1.50 & 2.20 & 2.85 & 2.10 & 2.60 & 3.35 \\
\hline 7,8 & 0.078 & 4.6 & 1.90 & 2.45 & 3.00 & 2.50 & 2.90 & 3.50 \\
\hline 9,11 & 0.023 & 6.8 & 1.55 & 2.25 & 2.90 & 2.20 & 2.70 & 3.40 \\
\hline 10 & 0.017 & 7.7 & 1.50 & 2.20 & 2.85 & 2.10 & 2.60 & 3.35 \\
\hline
\end{tabular}


Assuming that at time $t$ all elements have deterioration reliability indices exactly equal to their target $\beta_{D E i}^{T}$ according to Table 2, the probability of each system deterioration state, $\operatorname{Pr}\left[\Psi=\psi_{i}\right]$, $i=1,2, \ldots, 2048$, is computed. The true system deterioration reliability index $\beta_{D S}$ of the structural system in Figure 6 with the $\beta_{D E i}^{T}$ as given in Table 2 is then computed by Equation (1). The results are summarized in Table 3. Also listed in the table in parentheses are true system reliability indices obtained when using $\beta_{D E i}^{T}$ as determined by the current simplistic method, which disregards the statistical dependence between deterioration failures.

Table 3. Resulting system deterioration reliability indices for the validation structural system (in parentheses: values obtained with the existing simplistic approach).

\begin{tabular}{llll}
\hline Target $\beta_{D S}^{T}$ & \multicolumn{3}{c}{$\beta_{D S}$} \\
\cline { 2 - 4 } & $\rho_{M}=0.0$ & $\rho_{M}=0.3$ & $\rho_{M}=0.6$ \\
\hline 3.7 & $3.5(1.8)$ & $3.4(1.4)$ & $3.5(1.3)$ \\
4.2 & $4.1(3.8)$ & $4.0(3.0)$ & $4.0(2.5)$ \\
\hline
\end{tabular}

As observed in Table 3, the proposed use of the idealized systems leads in all investigated cases to a system deterioration reliability index that is close to but somewhat lower than the system deterioration target reliability index. More striking, however, is the significant improvement relative to the existing simplistic method. This is due to the approximate accounting of the dependence between the deterioration failure events of the structural elements by use of the equivalent Daniels systems.

\section{Numerical investigation of influencing factors}

The proposed model is applied to investigate the influence of the main input parameters. The following base case is considered: $\beta_{\overline{D S}}=4.4 ; \beta_{D S}^{T}=3.7 ; n=20 ; S E I_{i}=10^{-4}$ for $i=1, \ldots, n$; $\rho_{M}=0.4 ; L$ is Lognormal distributed with $\mu_{L}=1.0$ and c.o.v. $\delta_{L}=0.35 ; R_{i}$ are Normal distributed with c.o.v. $\delta_{R_{i}}=0.15$; all elements have ductile material behavior. These values of 
$\beta_{\overline{D S}}$ and $\beta_{D S}^{T}$ correspond to the case of a structure with large consequences of failure and with normal cost of safety measures against overload failures and large cost of safety measures against deterioration failures, see Table 1. Figure 7 presents the deterioration target reliability index $\beta_{D E i}^{T}$ for the elements as a function of the system parameters.
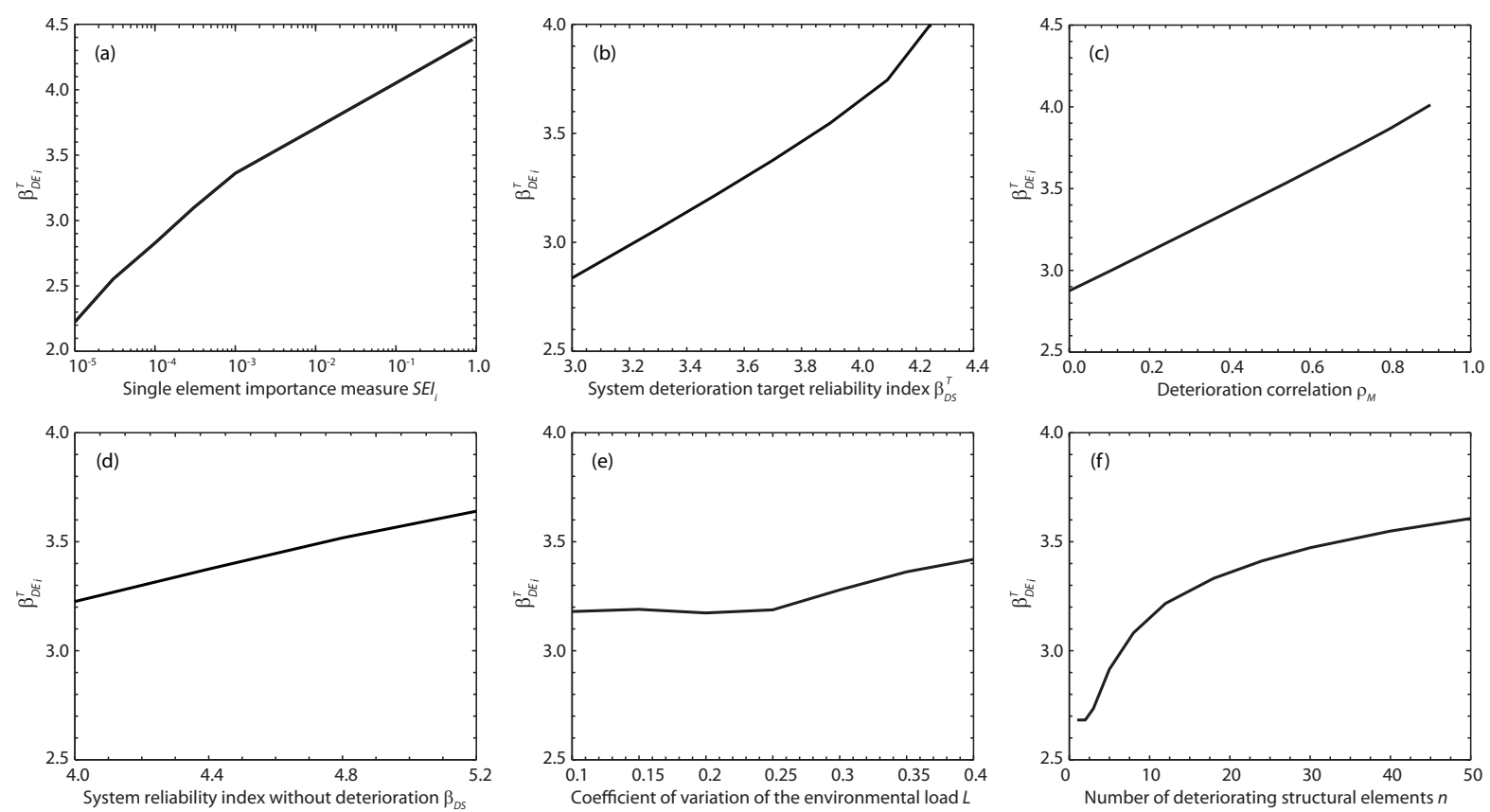

Figure 7. Target reliability indices as a function of various influencing parameters.

The results in Figure 7 allow identifying the main influencing parameters. As expected, the structural importance of the element, as expressed through the $S E I_{i}$, is a key parameter (Figure $7 \mathrm{a}$ ), as is the target reliability index for deterioration on the system level, $\beta_{D S}^{T}$ (Figure $7 \mathrm{~b}$ ). As confirmed by the numerical investigations presented earlier, the statistical dependence among deterioration safety margins has a strong influence on the system reliability (Figure $7 \mathrm{c}$ ). The resulting deterioration target reliability index for $\rho_{M}=0.4$ is $\beta_{D E i}^{T}=3.4$ as opposed to 
$\beta_{D E i}^{T}=2.9$ for the case of no correlation $\rho_{M}=0.0$. This demonstrates that statistical dependence among deterioration failure events of the elements must be considered when determining the target reliability indices of redundant systems.

The reliability of the intact structure $\beta_{\overline{D S}}$ has a moderate influence on $\beta_{D E i}^{T}$ (Figure $7 \mathrm{~d}$ ). $\beta_{D E i}^{T}$ increases with increasing $\beta_{\overline{D S}}$, which is due to the influence of $\beta_{\overline{D S}}$ on the $S E I_{i}$, Equation (7); for fixed value of the $S E I_{i}$, the probability of collapse given deterioration failure of element $i$ increases with increasing $\beta_{\overline{D S}}$. The influence of $\delta_{L}$, the c.o.v. of the annual maximum load on the structure, is low (Figure 7e), which is fortunate, since this indicates that assumptions regarding the overload failure mode of the structure are not critical when determining $\beta_{D E i}^{T}$.

Figure $7 \mathrm{f}$ demonstrates that $\beta_{D E i}^{T}$ increases with increasing number of elements. This fact may seem counter-intuitive but is due to the fact that the element structural importance is held constant in the numerical investigation shown in Figure 7d. In reality, structures with more elements tend to exhibit higher degrees of redundancy, thus having lower $S E I_{i}$. To account for this effect, Figure 8 presents $\beta_{D E i}^{T}$ for systems with varying degrees of redundancy. $\beta_{D E i}^{T}$ is shown as a function of $S E I_{i}$, whereby the parameter describing the system size is held constant as $k=5$. The number of elements is then computed as $n=k n_{i}$, with $n_{i}$ being a function of the $S E I_{i}$. As an example, for $S E I_{i}=10^{-3}$ it follows that $n_{i}=2$ and thus $n=10$, whereas for $S E I_{i}=10^{-5}, n_{i}=8$ and $n=40$. 


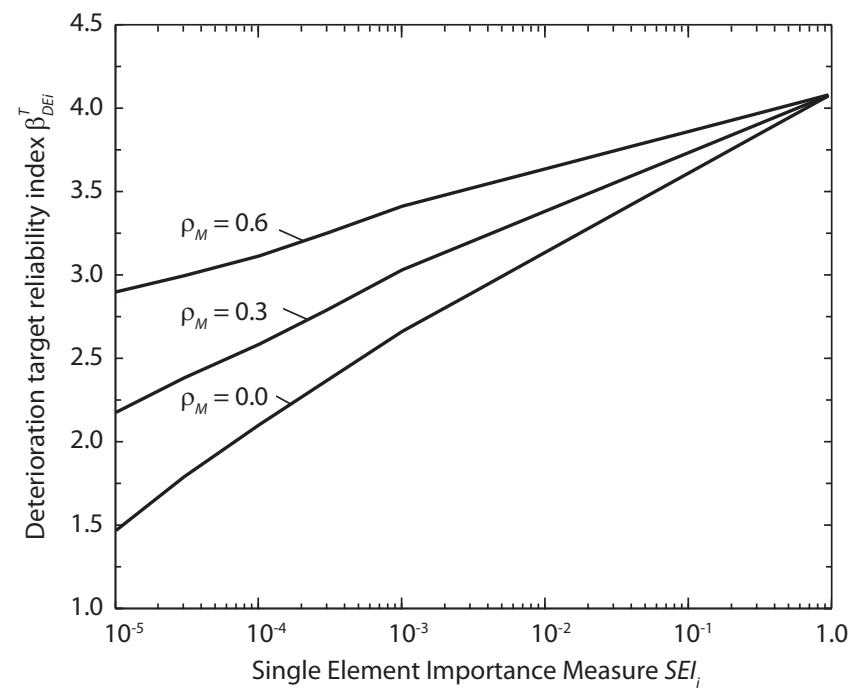

Figure 8. Target reliability indices obtained according to the proposed Daniels system model for example systems with varying number of elements and corresponding $S E I_{i}$.

\section{Concluding remarks}

As illustrated by the numerical examples in this paper, system effects, i.e., the joint effect of several deterioration failures on the structural integrity, are relevant when determining target reliability indices for deteriorating elements in redundant structural systems. However, a full analysis of the system, which includes system reliability assessments for all combinations of deterioration failures, is impractical for general structures. For this reason, highly simplified system models have been used in the past to describe the effect of an element failure on the integrity of the structure. These models do not represent the deterioration system effects adequately and are not suitable for redundant structures. To account for the system effects in determining acceptance criteria for individual deteriorating elements, this paper proposes using idealized Daniels systems to represent the deteriorating elements in the structural system. This is an idealization of the true system, which facilitates computation while capturing the overall 
characteristics of the structural system, including its redundancy (load-sharing among elements), and the influence of statistical dependence among deterioration failures on the effective redundancy. Indicators for the structural importance of the system elements that have been applied by previous approaches, such as the SEI, are used to define the characteristics of the idealized Daniels systems. As demonstrated by the validation example, the proposed model represents a significant improvement over current methods.

The proposed model is based on a number of idealizations and assumptions. In applying the model, it must be checked whether these are justified, or whether the model must be extended. Future research should be directed towards investigating applications for which these assumptions do not hold. Two idealizations/assumptions of the model are deemed critical for a number of applications: (a) the representation of deterioration by a two-state random variable, which neglects that deterioration occurs gradually, and (b) disregard of progressive deterioration failures. Concerning (a), future research efforts should be directed towards identifying deterioration limit state functions which best represent the effect of deterioration on the system reliability. It is noted that the current practice for defining deterioration failure is often conservative, in particular for fatigue, where structural elements at failure still retain most of their capacity. Concerning (b), progressive deterioration might be accounted for within the existing model framework by assigning high correlation coefficients and an increased probability of deterioration failure of the individual elements. Alternatively, the structural elements that are jointly affected by the progressive deterioration mechanism might be considered as a single (macro-)element in the system model. 


\section{Acknowledgements}

This work was partially supported by the Swiss National Science Foundation (SNF) through grant PA002-111428.

\section{References}

Ciampoli, M. (1998), Time dependent reliability of structural systems subject to deterioration, Computers \& Structures, 67(1-3), 29-35.

Daniels, H.E. (1945), The statistical theory of the strength of bundles of threads, Part I. Proc. Roy. Soc., A, 1945. 183: p. 405-435.

Ditlevsen, O. and H.O. Madsen (1996), Structural Reliability Methods. 1996: John Wiley \& Sons.

EC3 (1992), Eurocode 3 - Design of Steel Structures. ENV 1993-1-1. 1992.

Estes, A. C., and D. M. Frangopol (1999), Repair Optimization of Highway Bridges Using System Reliability Approach, Journal of Structural Engineering, Trans. ASCE, 125(7): 766-775.

Frangopol, D. M., M. J. Kallen, and J. M. van Noortijk (2004), Probabilistic models for life-cycle performance of deteriorating structures: review and future directions, Progress in Structural Engineering and Materials, 6: $197-212$.

Gharaibeh, E. S., D. M. Frangopol, and T. Onoufriou (2002), Reliability-based importance assessment of structural members with applications to complex structures, Computers \& Structures, 80(12): 11131131.

Gollwitzer, S. and R. Rackwitz (1990), On the Reliability of Daniels Systems. Structural Safety, 7: 229-243.

Hohenbichler, M. and R. Rackwitz (1982), First-order concepts in system reliability. Structural Safety, 1(3): 177188.

HSE (2002), Target levels for reliability-based assessment of offshore structures during design and operation. Offshore Technology Report, ed. H.a.S.E. (UK). 2002: HSE Books.

HSE (2006), Floating production system - JIP FPS mooring integrity. 2006, Research Report 444, Health and Safety Executive, UK.

JCSS (2006), Probabilistic Model Code. 2006, Joint Committee on Structural Safety (JCSS), internet publication: www.jcss.ethz.ch.

Kirkemo, F. (1990), Probabilistic strategy increases jacket in-service inspection efficiency, Offshore, 50(12), 4647.

Li, C. Q. (1995), Computation of the failure probability of deteriorating structural systems, Computers \& Structures, 56(6): 1073-1079. 
Lotsberg I, Kirkemo F. (1989). A Systematic Method for Planning In-Service Inspections of Steel Offshore Structures. Proc. OMAE 89, The Hague, The Netherlands.

Melchers, R. E. (2001), Assessment of Existing Structures-Approaches and Research Needs, Journal of Structural Engineering, Trans. ASCE, 127(4): 406-411.

Moan, T., (2005) Reliability-based management of inspection, maintenance and repair of offshore structures. Structure and Infrastructure Engineering, 2005. 1(1): 33-62.

Mori, Y., and B. R. Ellingwood (1993), Reliability-based service-life assessment of aging concrete structures, Journal of Structural Engineering, Trans. ASCE, 119(5): 1600-1621.

NORSOK (1998), Design of Steel Structures. Vol. Standard N-004, revision 1. 1998.

Rackwitz, R. (2000), Optimization - the basis of code-making and reliability verification. Structural Safety, 2000. 22(1): p. 27-60

Ronalds, B.F., et al. (2003). Jacket Reliability Design Considering Interacting Limit States. in Proc. 22nd International Conference on Offshore Mechanics and Arctic Engineering. Cancun, Mexico: ASME.

Stahl, B., et al. (2000), Acceptance Criteria for Offshore Platforms. Journal of Offshore Mechanics and Arctic Engineering, Trans. ASME, 2000. 123(3): 153-156.

Stewart, M. G., and D. V. Val (1999), Role of Load History in Reliability-Based Decision Analysis of Aging Bridges, Journal of Structural Engineering, Trans. ASCE, 125(7): 776-783.

Stewart, M. G., and D. V. Val (2003), Multiple Limit States and Expected Failure Costs for Deteriorating Reinforced Concrete Bridges, Journal of Bridge Engineering, Trans. ASCE, 8(6): 405-415.

Straub, D. and M.H. Faber (2005a), Risk Based Acceptance Criteria for Joints Subject to Fatigue Deterioration. Journal of Offshore Mechanics and Arctic Engineering, Trans. ASME, 2005. 127(2): 150-157.

Straub, D. and M.H. Faber (2005b), Risk based inspection planning for structural systems. Structural Safety, 2005. 27(4): 335-355

Vrouwenvelder, T. (2004). Spatial correlation aspects in deterioration models. in Proc. 2nd International Conference on Lifetime-Oriented Design Concepts. 2004. Bochum, Germany. 


\section{List of Figures}

Figure 1: Idealized structural system under external load. Case a) brittle element behavior (original Daniels system), b) ductile element behavior.

Figure 2: Failure probability of Daniels system as a function of the number of elements failed due to deterioration.

Figure 3: System deterioration reliability index as a function of number of elements (left chart), element deterioration reliability index (middle chart), and correlation among deterioration limit states (right chart).

Figure 4: The equivalent system for element $i$. All $n_{i} \cdot k$ elements have independent identically distributed capacities $R_{i, j}$ relative to overload failure. System failure occurs if any of the $k$ subsystems fails.

Figure 5: Algorithm for establishing the equivalent systems.

Figure 6: Structural system for model validation.

Figure 7: Target reliability indices as a function of various influencing parameters.

Figure 8: Target reliability indices obtained according to the proposed Daniels system model for example systems with varying number of elements and corresponding $S E I_{i}$.

\section{List of Tables}

Table 1: Tentative target reliability indices $\beta^{T}$ for ultimate limit states and one year reference period, as recommended in JCSS (2006).

Table 2: Resulting deterioration target reliability indices for the validation structure.

Table 3: Resulting system deterioration reliability indices for the validation structural system (in parentheses: values obtained with the existing simplistic approach). 